\title{
Transmission of cytomegalovirus via breast milk in low birth weight and premature infants: a systematic review and meta-analysis
}

\author{
Xiaolin Hu, Wei Hu, Xuan Sun, Ling Chen and Xiaoping Luo*
}

\begin{abstract}
Background: This study aimed to investigate the transmission of cytomegalovirus (CMV) via breast milk in low birth weight (LBW) and premature infants and its effects.

Methods: PubMed, Medline, Cochrane Library, and Embase were searched for studies (without language and time restriction) published before March 27, 2020, that examined the effect of CMV transmitted by breast milk on LBW and premature infants. The rates of breast milk-acquired CMV infection, CMV-related symptoms, and CMV-related sepsislike syndrome (CMV-SLS) in LBW and premature infants were pooled from each study.

Results: Eighteen studies with 1920 LBW and premature infants were included. The pooled CMV infection rate from breast milk for infants fed untreated breast milk was significantly higher than those fed frozen breast milk [19.3, 95\% confidence interval $(\mathrm{Cl})=11.8-29.9 \%$ vs. $13.5,95 \% \mathrm{Cl}=8.0-22.0 \%, P<0.01)$. Similarly, the pooled CMV infection rate for infants fed untreated breast milk was significantly higher than those with mixed feeding $(P<0.0001)$. The mixed feeding group had a significantly lower rate of CMV-related symptoms than the other groups $(2.4 \%, P<0.01)$.

Conclusions: These findings suggested a higher CMV infection rate in LBW or premature infants fed untreated breast milk than other feeding groups. Studies on the long-term outcomes of CMV infection transmitted from breast milk are needed to address the optimal feeding practice.
\end{abstract}

Keywords: Breast milk, Cytomegalovirus, Preterm infant, Infection transmission, meta-analysis

\section{Background}

Cytomegalovirus (CMV) is a member of the beta-herpes virus subfamily and causes serious clinical consequences in premature infants $[1,2]$, including respiratory failure, neutropenia, thrombocytopenia, hepatomegaly, and septic syndromes [3, 4]. Over the past few years, breast milk from seropositive mothers has been considered the main source of postnatally acquired CMV infection in preterm infants $[5,6]$. CMV infection is often diagnosed by polymerase chain reaction (PCR), cell culture, and

*Correspondence: xpluo@tjh.tjmu.edu.cn

Pediatric Department, Tongji Hospital, Tongji Medical Collage, Huazhong

University of Science and Technology, Wuhan, China identification of CMV-specific antibodies or intracellular viral proteins $[2,6]$.

Almost $96 \%$ of the seropositive mothers have CMV reactivation, defined as shedding of viable virus or the presence of CMV deoxyribonucleic acid (DNA) in breast milk [7]. Previous studies revealed that the transmission rate of CMV from CMV-positive breast milk to preterm infants ranges from 37 to $87 \%[5,7,8]$. Moreover, preterm infants were at higher risk of CMV infection than full-term newborns [6]. In addition, poor outcomes such as abnormal laboratory findings to a sepsis-like syndrome and even neurologic sequelae were observed in preterm infants $[9,10]$. 
A previous meta-analysis [11] reported that CMVrelated sepsis-like syndrome (CMV-SLS) is relatively rare in infants with breast milk-acquired CMV infection. Still, recently, several studies $[12,13]$ on breast milk-acquired CMV infection in low birth weight (LBW) or preterm infants provided new evidence on this topic. In addition, some studies $[13,14]$ reported a strong association between breast milk-acquired CMV and CMV-SLS in LBW and premature infants. Thus, the most recent published studies make it possible to complete a new systematic review and meta-analysis with more statistical power. Therefore, a meta-analysis was conducted to investigate the effects of transmission of CMV via breast milk in LBW and premature infants.

\section{Methods}

\section{Literature search}

PubMed, Medline, Cochrane Library, and Embase were searched for relevant studies on the research topic published before March 27, 2020. The individual and combined key words such as "breastfeeding", "breast milk", "premature", "preterm", "low birth weight infants", "cytomegalovirus", and "CMV" were used for searching the literature. The detailed search strategy can be found in Supplementary Table 1 . The search terms were kept as broad as possible to identify relevant publications. In addition, in order to include more potential studies, bibliographies of all relevant studies and reviews identified were searched, and also Google Scholar was searched for relevant studies. This current study was conducted according to the Preferred Reporting Items for Systematic Reviews and Meta-analysis guidelines (PRISMA) statement [15].

\section{Eligibility criteria}

Studies that investigated the effects of transmission of CMV infection via breast milk in LBW and premature infants were included. The inclusion criteria were 1) observational studies reporting the results of transmission of CMV infection via breast milk, 2) study subjects were LBW and premature infants, 3) data could be obtained from the original studies, 4) studies published in English, and 5) studies with more detailed information were selected if the population was reported in duplicate.

Reviews, case reports, comments, animal experiments, studies in languages other than English, and unavailable studies were excluded from the analysis.

\section{Data extraction and definition}

All relevant articles from the databases mentioned above were screened independently by two authors (XH and WH) to decide whether the studies could be included in the full-text analysis. The data and necessary information of the relevant studies were extracted using a standardized form independently by two reviewers (XH and $\mathrm{WH})$, and a consensus was reached on all items by a discussion with a third reviewer (XL). Information such as study characteristics (e.g., author and year of publication, country, sample size, and study design), patient characteristics (e.g., weight and gestational age at birth, sex of infants, and nationality), CMV infection characteristics (e.g., methods for diagnosing congenital CMV infection and postnatal CMV infection among infants), breast milk characteristics (e.g., pasteurization, freezing, and no treatment), and infant characteristics (e.g., numbers of infants who acquired postnatal CMV and outcomes, birth weight, and corrected gestational age at the onset of CMV infection) were extracted from each study.

Congenital CMV infection was defined as viral culture or CMV nucleic acid testing positive in blood or urine samples obtained within 2 weeks after birth. Postnatal $\mathrm{CMV}$ infection was defined as a positive congenital CMV positive and with a previously documented negative result. Infants born before 37 weeks gestation are considered premature.

\section{Quality scoring of studies}

The two reviewers mentioned above (XH and $\mathrm{WH}$ ) assessed the methodological strength of all included studies independently using the Newcastle-Ottawa Scale (NOS) [16], a procedure for independently assessing the methodological quality of observational studies. The NOS includes three categories: 1) patient selection (three items), 2) comparability of two study arms (two items), and 3) assessment of the outcomes (two items).

Studies were given a maximum of one point for each item within the selection and exposure categories and a maximum of two points for comparability. Studies were graded based on an ordinal scoring scale, and the score ranged from 2 to 9 stars; 2 to 5 stars was considered poor quality and 6-9 stars high quality. In order to provide more information on the quality assessment, we also used the Critical Appraisal Skills Programme (CASP) cohort checklist for the included studies (available at: https://casp-uk.net).

\section{Statistical analysis}

The estimated rates of breast milk-acquired CMV infection, CMV-related symptoms, and CMV-SLS in LBW were extracted from each included study. The estimated rates of breast milk-acquired CMV infection, CMVrelated symptoms, and CMV-SLS in LBW and premature infants were pooled by rates and relevant $95 \%$ confidence intervals (CIs). Inverse variance methods with randomeffects were conducted to pool the results of included studies. The standard heterogeneity test, $I^{2}$ statistic, 
was used to assess the consistency of the effect sizes. It indicates the percentage of variability in effect estimates because of true between-study variance rather than within-study variance. Heterogeneity was categorized into with and without significant heterogeneity according to the values of $I^{2}$ by $\geq 50$ and $<50 \%$ [17], respectively. In order to explore the sources of heterogeneity, all enrolled studies were sequentially excluded to demonstrate the overall impact of individual studies, where $I^{2}>50 \%$ indicated significant heterogeneity. We also conducted random-effects meta-regression analyses to assess the impact of covariates on infection effects. Publication bias was assessed by Begg's rank correlation [18] and Egger's weighted regression methods [19]. Review Manager (version 5.3, The Cochrane Collaboration, Oxford, UK) was used for statistical analyses. The Begg's and Egger's tests were assessed by STATA 15.0 (Stata Corporation, College Station, TX, USA). A $P$ value of $<0.05$ was statistically considered significant for all analyses.

\section{Results}

\section{Study selection}

The study selection process is presented in Fig. 1. The systematic literature search yielded a total of 826 studies. Of these, 352 were excluded due to duplications, and the abstracts of 418 were initially reviewed. After careful reviewing, the full-length manuscripts of 56 studies were obtained. Following the inclusion and exclusion criteria, 18 studies [7, 12-14, 20-33] were finally included for data extraction and meta-analysis after excluding one study that used the same population [34].

\section{Study characteristics}

The 18 studies included in this review included a total of 1920 LBW and premature infants. Two studies were divided into two studies according to the treatment method of the milk. The included studies were published between 2001 and 2019. The number of infants in each study ranged from 7 to 539 . Most studies, except one, were prospective cohort studies [33]. Four of the included studies were conducted in Germany [12, 22, 26, 30], two in the United States of America [14, 31], two each in Brazil [13, 21], Japan [20, 33], Sweden [26, 32], Taiwan [21, 29], and Italy [28], and one each in Canada [23], Israel [25], and Korea [33]. Of the included studies, 17 used PCR, four used rt-PCR (real-time polymerase chain reaction), and one used immunofluorescence for CMV detection in infants. The inclusion criteria for infants were based on gestational age (GA, weeks) and/ or body weight (BW, grams) of the infants. Most studies

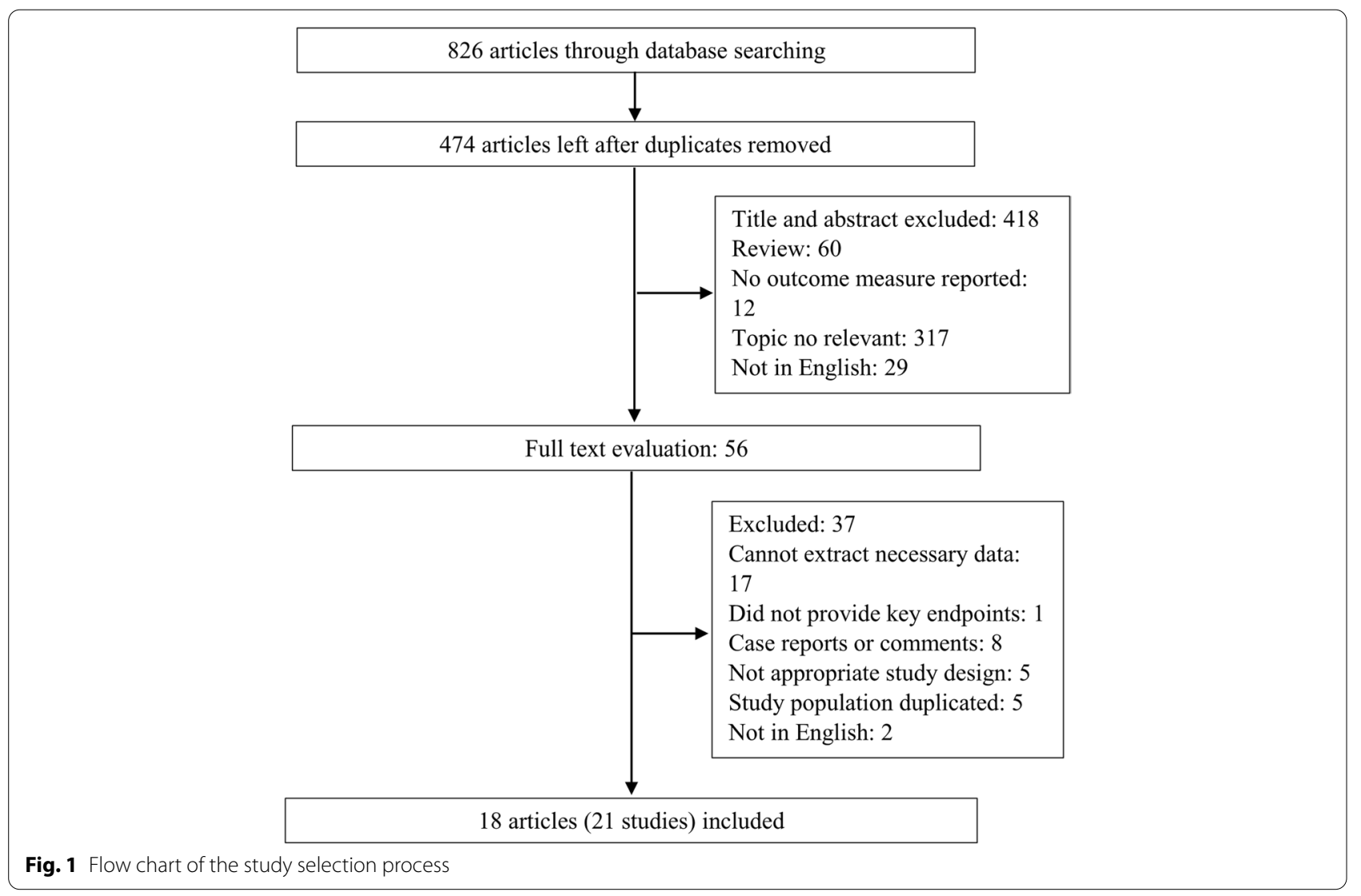


set $1500 \mathrm{~g}$ as the cut-off value for BW (ranged from 1000 to $1710 \mathrm{~g}$ of the included studies) and 32 weeks as the criteria for GA (ranged from 28 to 35 weeks). Most studies defined 21 days as the cutoff for testing for postnatally acquired CMV. The infants in 12 included studies were fed untreated breast milk, and in six studies were fed breast milk that was frozen at $-18^{\circ} \mathrm{C}$ to $-20^{\circ} \mathrm{C}$ for $>24$ or $72 \mathrm{~h}$. In seven studies, the infants were fed untreated breast milk in combination with frozen breast milk and/ or pasteurized breast milk from donors. Seventeen studies reported the means or median of BW of infants, which ranged from 748 to $1300 \mathrm{~g}$. The characteristics of the included studies and participants are summarized in Supplementary Tables 1 and 2. As shown in Supplementary Fig. 1, a total of 1598 mothers in the included studies were reported to be serologically CMV positive (IgG or IgM, or the combination of IgG and IgM). The pooled CMV positive rate of the included mothers was $77.6 \%$ $\left(95 \% \mathrm{CI}=66.0-86.0 \%, I^{2}=24 \%\right)$.

\section{Quality assessment of the studies}

All eligible studies were of high quality ( $\geq 6$ stars). Some of the included studies did not conduct adequacy of follow-up of their cohorts. Meanwhile, most studies enrolled outpatients, and the representativeness, therefore, was insufficient. The NOS scores for the eligible studies are presented in Supplementary Table 3. Similarly, CASP also indicated the high quality of all the included studies.

\section{CMV transmission to the preterm infant}

Of the 12 studies that reported infants fed untreated breast milk, 1205 infants were included. The rate of CMV infection in each study ranged from 0 to $64 \%$. The pooled rate of CMV infection for infants fed untreated breast milk was $19.3 \%(95 \% \mathrm{CI}=11.8-29.9 \%, P<0.01)$, and showed no significant heterogeneity $\left(I^{2}=37 \%\right)$. For infants fed frozen breast milk, 52 CMV-positive infants were included. The pooled CMV infection rate (13.5, $95 \% \mathrm{CI}=8.0-22.0 \%, P<0.01, I^{2}=46 \%$ ) was significantly lower than that of infants fed untreated breast milk (overall $P<0.01$ ). Seven studies with 768 infants reported the result on infants fed mixed milk. The pooled CMV infection rate $\left(9.1,95 \% \mathrm{CI}=4.2-18.5 \%, P<0.01, I^{2}=30 \%\right)$ was similar to the infants who were fed frozen breast milk and were significantly lower than that of infants fed untreated breast milk $(P<0.01)$. The pooled CMV infection rate for all infants was $14.4 \%(95 \% \mathrm{CI}=10.1-20.2 \%, P<0.01$, $I^{2}=40 \%$ ). The detailed data and funnel plot are shown in Fig. 2 and Supplementary Fig. 2.

\begin{tabular}{|c|c|c|c|c|c|c|c|c|c|c|}
\hline Study name & Subgroup within study & Event rate & Lower limit & Upper limit & P-valu & & & & & \\
\hline Yasuda et.al., 2003 & Frozen breast milk & 0.100 & 0.033 & 0.268 & 0.000 & 一— & & & & \\
\hline Jim et.al., 2004 & Frozen breast milk & 0.150 & 0.069 & 0.296 & 0.000 & $\longrightarrow$ & & & & \\
\hline Jim et.al., 2009 & Frozen breast milk & 0.348 & 0.184 & 0.557 & 0.151 & & - & & & \\
\hline Buxmann et.al., 2009 & Frozen breast milk & 0.143 & 0.061 & 0.300 & 0.000 & $\longrightarrow$ & & & & \\
\hline Yoo et.al., 2015 c & Frozen breast milk & 0.084 & 0.058 & 0.119 & 0.000 & - & & & & \\
\hline Omarsdottir et.al., 2015 c & Frozen breast milk & 0.086 & 0.028 & 0.234 & 0.000 & $\rightarrow-$ & & & & \\
\hline Overall frozen breast milk & & 0.135 & 0.080 & 0.220 & 0.000 & $\rightarrow-$ & & & & \\
\hline Mussi-Pinhata et.al., 2004 a & Mixed & 0.174 & 0.089 & 0.311 & 0.000 & $\longrightarrow$ & & & & \\
\hline Doctor et.al., 2005 & Mixed & 0.066 & 0.025 & 0.162 & 0.000 & $-\square-$ & & & & \\
\hline Omarsdottir et.al., 2007 & Mixed & 0.286 & 0.072 & 0.673 & 0.273 & & & & & \\
\hline Hayashi et.al., 2011 & Mixed & 0.045 & 0.006 & 0.261 & 0.003 & $\rightarrow-$ & & & & \\
\hline Yoo et.al., $2015 d$ & Mixed & 0.008 & 0.000 & 0.115 & 0.001 & 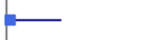 & & & & \\
\hline Omarsdottir et.al., 2015 a & Mixed & 0.065 & 0.016 & 0.224 & 0.000 & $\rightarrow-$ & & & & \\
\hline Overall mixed & & 0.091 & 0.042 & 0.185 & 0.000 & $\rightarrow-$ & & & & \\
\hline Hamprecht et.al., 2001 & Untreated breast milk & 0.367 & 0.274 & 0.471 & 0.012 & & & & & \\
\hline Mussi-Pinhata et.al., 2004 b & Untreated breast milk & 0.265 & 0.161 & 0.405 & 0.002 & & & & & \\
\hline Miron et.al., 2005 & Untreated breast milk & 0.057 & 0.022 & 0.143 & 0.000 & $\rightarrow-$ & & & & \\
\hline Capretti et.al., 2009 & Untreated breast milk & 0.145 & 0.077 & 0.256 & 0.000 & $\longrightarrow$ & & & & \\
\hline Mehler et.al., 2014 & Untreated breast milk & 0.647 & 0.404 & 0.832 & 0.232 & & & & & \\
\hline Martins-Celini et.al., 2016 & Untreated breast milk & 0.165 & 0.098 & 0.263 & 0.000 & & & & & \\
\hline Bapistella et.al., 2018 & Untreated breast milk & 0.023 & 0.006 & 0.087 & 0.000 & - & & & & \\
\hline Donalisio et.al., 2018 & Untreated breast milk & 0.333 & 0.158 & 0.571 & 0.166 & & & & & \\
\hline Lopes et.al., 2018 & Untreated breast milk & 0.340 & 0.255 & 0.436 & 0.001 & & - & & & \\
\hline Mukhopadhyay et.al., 2018 & Untreated breast milk & 0.186 & 0.131 & 0.258 & 0.000 & - & & & & \\
\hline Patel et.al., 2019 & Untreated breast milk & 0.072 & 0.052 & 0.100 & 0.000 & $=$ & & & & \\
\hline \multirow[t]{2}{*}{ Overall untreated breast milk } & & 0.193 & 0.118 & 0.299 & 0.000 & $\longrightarrow$ & & & & \\
\hline & & & & & & 0.2 & 0.4 & 0.6 & 0.8 & 1 \\
\hline
\end{tabular}




\section{CMV-related symptoms}

The included studies mainly reported CMV-related symptoms such as thrombocytopenia, neutropenia, hepatitis, hepatosplenomegaly, and elevated liver enzymes, sequentially. The pooled symptom rates for infants fed untreated breast milk, frozen breast milk, and mixed feeding were $8.3 \%(95 \% \mathrm{CI}=4.8-14.0 \%$, $P<0.01$ ), $8.0 \%$ (95\% CI $=5.0-12.6 \%, P<0.01)$, and $3.7 \%$ (95\% CI $=1.5-8.6 \%, P<0.01)$, respectively. The pooled overall rate was $7.5 \%(95 \% \mathrm{CI}=5.1-10.7 \%, P<0.01)$. The mixed group had a significantly lower rate of symptoms than the other groups $(P<0.01)$. All pooled results demonstrated no significant heterogeneity (all $\left.I^{2}<50 \%\right)$. More detailed data were summarized in Fig. 3.

\section{CMV-SLS}

Twenty-two studies with 2362 patients provided data on CMV-SLS. The pooled rate or infants fed untreated breast milk was $4.2 \%(95 \% \mathrm{CI}=2.0-8.4 \%, P<0.01)$ and showed lower heterogeneity $\left(I^{2}=30 \%\right)$. The detailed data are presented in Fig. 4. The mixed feeding group demonstrated a significantly lower rate than the other two groups $(P<0.01)$.

\section{Meta-regression analyses}

Meta-regression analyses were performed for the types of milk, NOS score, means of gestational ages, and study design. The types of milk were significantly associated in a gradient fashion $(P<0.0001$, Supplementary Fig. 3$)$. However, no significant gradient associations were observed for the other outcomes.

\section{Publication bias}

No potential publication bias among the included trials was observed according to Begg's rank correlation analysis and Egger's weighted regression analysis (all $P>0.05$, Supplementary Table 4).

\section{Discussion}

In the current meta-analysis, 21 studies with a total of 1920 LBW and premature infants were included. All included studies were of relatively high quality. The pooled rate for infants fed untreated and frozen breast milk was 19.3 and $13.5 \%$, respectively. LBW or preterm infants in the untreated breast milk group were associated with a significantly higher CMV infection rate via breast milk when compared to other feeding groups.

Comparing with the previous meta-analysis [11], we included more studies (11 vs. 21) with more infants.

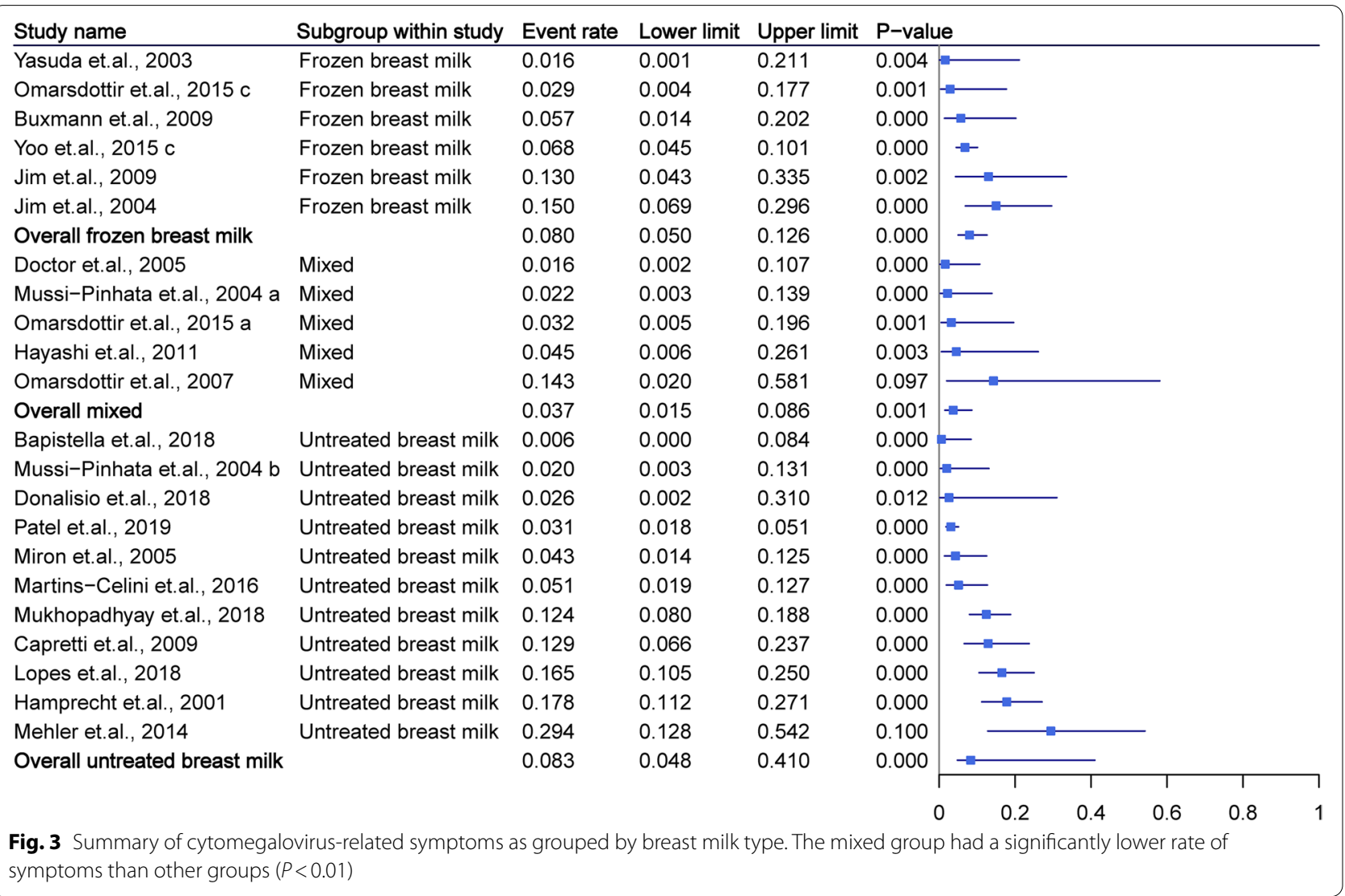




\begin{tabular}{|llllll|}
\hline Study name & Subgroup within study & Event rate & Lower limit & Upper limit & P-value \\
\hline Yasuda et.al., 2003 & Frozen breast milk & 0.016 & 0.001 & 0.211 & 0.004 \\
Jim et.al., 2009 & Frozen breast milk & 0.125 & 0.053 & 0.267 & 0.000 \\
Jim et.al., 2004 & Frozen breast milk & 0.087 & 0.022 & 0.289 & 0.001 \\
Buxmann et.al., 2009 & Frozen breast milk & 0.014 & 0.001 & 0.187 & 0.003 \\
Yoo et.al., 2015 c & Frozen breast milk & 0.022 & 0.010 & 0.045 & 0.000 \\
Overall frozen breast milk & & 0.045 & 0.016 & 0.120 & 0.000 \\
Mussi-Pinhata et.al., 2004 a Mixed & Mixed & 0.011 & 0.001 & 0.149 & 0.001 \\
Doctor et.al., 2005 & Mixed & 0.016 & 0.002 & 0.107 & 0.000 \\
Omarsdottir et.al., 2007 & Mixed & 0.143 & 0.020 & 0.581 & 0.097 \\
Hayashi et.al., 2011 & Mixed & 0.016 & 0.001 & 0.268 & 0.008 \\
Omarsdottir et.al., 2015 a & & 0.028 & 0.010 & 0.079 & 0.004 \\
Overall mixed & Untreated breast milk & 0.044 & 0.017 & 0.112 & 0.000 \\
Hamprecht et.al., 2001 & Untreated breast milk & 0.010 & 0.001 & 0.141 & 0.001 \\
Mussi-Pinhata et.al., 2004 b & Untreated breast milk & 0.014 & 0.002 & 0.094 & 0.000 \\
Miron et.al., 2005 & Untreated breast milk & 0.048 & 0.016 & 0.140 & 0.000 \\
Capretti et.al., 2009 & Untreated breast milk & 0.353 & 0.168 & 0.596 & 0.232 \\
Mehler et.al., 2014 & Untreated breast milk & 0.038 & 0.012 & 0.111 & 0.000 \\
Martins-Celini et.al., 2016 & Untreated breast milk & 0.006 & 0.000 & 0.084 & 0.000 \\
Bapistella et.al., 2018 & Untreated breast milk & 0.026 & 0.002 & 0.310 & 0.012 \\
Donalisio et.al., 2018 & Untreated breast milk & 0.097 & 0.053 & 0.171 & 0.000 \\
Lopes et.al., 2018 & Untreated breast milk & 0.041 & 0.019 & 0.089 & 0.000 \\
Mukhopadhyay et.al., 2018 & Untreated breast milk & 0.013 & 0.006 & 0.029 & 0.000 \\
Patel et.al., 2019 & 0.042 & 0.020 & 0.084 & 0.000 \\
Overall untreated breast milk & & & & \\
& & & & & 0. \\
\hline
\end{tabular}

Fig. 4 Summary of cytomegalovirus sepsis-like syndrome as grouped by breast milk type. The mixed feeding group demonstrated a significantly lower rate than the other two groups $(P<0.01)$

However, similarly, the infants infected with CMV via breast milk were rare. CMV infection via breast milk does not usually occur in full-term infants due to the transmission of protective maternal antibodies. A previous study reported the transmission process of maternal antibodies, which start within 29 gestational weeks [35]. For LBW or premature infants, this transmission of antibodies from the mothers might be absent, putting the infants at high risk for CMV infection postnatally $[35,36]$. Therefore, premature infants, especially LBW infants, are associated with symptomatic and even severe CMV infection [37]. It has been reported that milk whey contains viable CMV virions due to different breast milk compartments. For the mothers who were CMV positive for milk, many of them were seropositive. A previous study has demonstrated the predominant role of cell-free virus transmission by breast milk [38]. While the risk of postnatal CMV infection is inversely proportional to $\mathrm{GA}$, several studies have reported a strong association between high CMV viral load in bone marrow cells and risk of transmission $[12,29]$, as well as an inverse correlation between CMV-specific IgG avidity in breast milk and CMV load [32].

Compared to term infants, preterm infants, especially high-risk preterm infants, have more immature organ development and are more likely to suffer from series of morbidities such as pulmonary, hematologic, or hepatic conditions. Thus, CMV infections likely cause damage to the developing organs, and its transmission via breast milk might be a cofactor in aggravating the clinical course of pre-existing diseases and neurological implications in certain preterm infants. CMV-SLS has been introduced to describe the severe symptoms associated with CMV infection in LBW or premature infants. In the current study, CMV transmission via breast milk that led to severe symptomatic infections (such as SLS) in infected infants was rare. The data on long-term sequelae, such as neurological and cognitive sequelae, and sensorineural hearing loss, was insufficient.

Breast milk is considered an optimal food for preterm infants due to its benefits in preventing many comorbidities such as necrotizing enterocolitis (NEC) and retinopathy of prematurity (ROP) and improving neurodevelopmental outcomes. NEC is an acute inflammatory reaction and is the leading gastrointestinal cause of morbidity and mortality in preterm infants [39]. Studies have shown that raw breast milk nutrition significantly reduces the risk of NEC [40]. However, the link between NEC and postnatal CMV is controversial [40]. Fresh breast milk contains beneficial factors, but the risk of 
CMV transmission to cause severe systematic symptoms cannot be neglected. In this meta-analysis, frozen breast milk or mixed feeding protocol were associated with a lower risk of CMV-related infection and injury. The prospective study conducted by Capretti et al. [28] reported that CMV infection though the mother's milk neither influenced short-term outcome or length of hospital stay nor did it cause long-term neurosensorial sequelae. Therefore, the benefits of fresh milk might overweight the risks related to CMV transmission for most LBW infants. However, currently, in the clinical settings, the breast milk feeding practices vary in different centers as many experts favor frozen breast milk for feeding in high-risk preterm infants. This perspective seems to be consistent with our study, which indicates frozen breast milk or mixed feeding are in priority for LBW or preterm infants. As comprehensive long-term follow up data on the impact of postnatal CMV infection have not yet established. The data from this kind of studies remain critical for future analysis and assists in constructing an optimal breast milk feeding practice in different countries based on eligible infants.

Although most included studies were of high quality (NOS $\geq 6$ scores), it is necessary to consider the limitations of the present meta-analysis when interpreting the results. Firstly, most of the studies included few participants. Due to the limited number of patients included in each study, it was difficult to perform subgroup or sensitivity analyses. Moreover, the representativeness of the target population might be weakened. Secondly, the inclusion criteria for each study varied. For example, most studies included infants with birth weight lower than $1200 \mathrm{~g}$ or GA earlier than 32 weeks, while others included infants lower than $1500 \mathrm{~g}$ or GA earlier than 35 weeks. Moreover, some studies only considered birth weight as the inclusion criteria. Thus, the definition for the targeted population might cause heterogeneity and reduce the stability of the results. Thirdly, the number of the included studies was limited, and most of them were conducted in western countries and focused on Caucasians. So, the results might be affected by the living factors, environmental factors, medical level, and genetic factors. Therefore, the results in the current setting can only partly annotate the associations. Fourthly, a relative effect measure such as the odds ratio would be more informative. However, due to the insufficient information provided by the included studies, we cannot implement that. Fifthly, potential language bias might exist because our literature searches considered only articles published in English. Lastly, more details on the duration of breastfeeding and the proportion of raw breast milk in the mixed feeding group require clarification. Their impact on the transmission of CMV via breast milk should be stratified in further investigation.

\section{Conclusions}

This meta-analysis provided pooled results based on 21 studies from 11 different regions or countries and summarized a data set of 1920 infants. The infants fed with untreated breast milk had a significantly higher CMV infection rate via breast milk in LBW or premature infants compared to other groups. The mixed feeding group had a significantly lower rate of CMV-related symptoms and CMV-SLS than the other groups. In the future, more original studies conducted in different counties and ethnic cultures are warranted to verify the results reported in this study.

\section{Abbreviations}

CMV: Cytomegalovirus; LBW: Low birth weight; CMV-SLS: CMV-related sepsislike syndrome; Cl: Confidence interval; PCR: Polymerase chain reaction; NOS: Newcastle-Ottawa Scale; Cls: Confidence intervals; GA: Gestational age; BW: Body weight; NEC: Necrotizing enterocolitis; ROP: Retinopathy of prematurity.

\section{Supplementary Information}

The online version contains supplementary material available at https://doi. org/10.1186/s12887-021-02984-7.

Additional file 1 : Supplementary Figure 1. Summary of cytomegalovirus infection rate in all included mothers.

Additional file 2 : Supplementary Figure 2. Funnel plot for the summary of overall cytomegalovirus infection rate.

Additional file 3 : Figure S3.

Additional file 4 : Supplementary Table 1. Literature search strategy.

Additional file $\mathbf{5}$ : Supplementary Table $\mathbf{2}$. Characteristics of study population.

Additional file $\mathbf{6}$ : Supplementary Table 2. Publication bias of summarized outcomes. Supplementary Table 3. Quality assessment of included studies by Newcastle-Ottawa Scale.

Additional file 7 : Supplementary Table 4. Publication bias of summarized outcomes.

\section{Acknowledgments}

The authors would like to thank all study participants who were enrolled in this study.

\section{Authors' contributions}

$\mathrm{XH}$ and $\mathrm{XL}$ carried out the studies, participated in collecting data, and drafted the manuscript. $\mathrm{XH}$ and $\mathrm{WH}$ performed the statistical analysis and critically for important intellectual content. XS and LC participated in the acquisition, analysis, or interpretation of data and drafted the manuscript. All authors read and approved the final manuscript.

\section{Funding}

This work was supported by the National Key R\&D Program of China (No. 2018YFC1002400 to XL) and Hubei Provincial Science Foundation (WJ2019M125).

\section{Availability of data and materials}

The datasets used and/or analyzed during the current study are available from the corresponding author on reasonable request. 


\section{Declarations}

Ethics approval and consent to participate

Not applicable.

\section{Consent for publication}

Not applicable.

\section{Competing interests}

The authors declare that they have no competing interests.

Received: 25 April 2021 Accepted: 20 October 2021

Published online: 22 November 2021

\section{References}

1. Ballard RA, Drew WL, Hufnagle KG, Riedel PA. Acquired cytomegalovirus infection in preterm infants. Am J Dis Child (1960). 1979;133:482-5.

2. Vochem M, Hamprecht K, Jahn G, Speer CP. Transmission of cytomegalovirus to preterm infants through breast milk. Pediatr Infect Dis J. 1998;17:53-8.

3. Hamprecht K, Maschmann J, Jahn G, Poets CF, Goelz R. Cytomegalovirus transmission to preterm infants during lactation. J Clin Virol. 2008:41:198-205.

4. Kurath S, Halwachs-Baumann G, Müller W, Resch B. Transmission of cytomegalovirus via breast milk to the prematurely born infant: a systematic review. Clin Microbiol Infect. 2010;16:1172-8.

5. Stagno S, Reynolds DW, Pass RF, Alford CA. Breast milk and the risk of cytomegalovirus infection. N Engl J Med. 1980;302:1073-6.

6. Dworsky M, Yow M, Stagno S, Pass RF, Alford C. Cytomegalovirus infection of breast milk and transmission in infancy. Pediatrics. 1983;72:295-9.

7. Hamprecht K, Maschmann J, Vochem M, Dietz K, Speer CP, Jahn G. Epidemiology of transmission of cytomegalovirus from mother to preterm infant by breastfeeding. Lancet (London, England). 2001;357:513-8.

8. From the $\mathrm{NIH}$. Breast milk and the risk of cytomegalovirus infection. Jama. $1981 ; 246: 2687$.

9. Yeager AS, Palumbo PE, Malachowski N, Ariagno RL, Stevenson DK. Sequelae of maternally derived cytomegalovirus infections in premature infants. J Pediatr. 1983:102:918-22

10. Paryani SG, Yeager AS, Hosford-Dunn H, Johnson SJ, Malachowski N, Ariagno $\mathrm{RL}$, et al. Sequelae of acquired cytomegalovirus infection in premature and sick term infants. J Pediatr. 1985;107:451-6.

11. Lanzieri TM, Dollard SC, Josephson CD, Schmid DS, Bialek SR. Breast milkacquired cytomegalovirus infection and disease in VLBW and premature infants. Pediatrics. 2013;131:e1937-45.

12. Mehler K, Oberthuer A, Lang-Roth R, Kribs A. High rate of symptomatic cytomegalovirus infection in extremely low gestational age preterm infants of 22-24 weeks' gestation after transmission via breast milk. Neonatology. 2014;105:27-32

13. Martins-Celini FP, Yamamoto AY, Passos DM, do Nascimento SD, Lima EV, Di Giovanni CM, et al. Incidence, risk factors, and morbidity of acquired postnatal cytomegalovirus infection among preterm infants fed maternal milk in a highly seropositive population. Clin Infect Dis. 2016;63:929-36.

14. Patel RM, Shenvi N, Knezevic A, Hinkes M, Bugg GW, Stowell SR, et al. Observational study of cytomegalovirus from breast milk and necrotising enterocolitis. Arch Dis Child Fetal Neonatal Ed. 2019;105:259-65.

15. Moher D, Shamseer L, Clarke M, Ghersi D, Liberati A, Petticrew M, et al. Preferred reporting items for systematic review and meta-analysis protocols (PRISMA-P) 2015 statement. Syst Rev. 2015:4:1.

16. Wells GA, Shea BJ, O'Connell D, Peterson J, Tugwell P. The Newcastle-Ottawa scale (NOS) for assessing the quality of non-randomized studies in metaanalysis; 2000

17. Higgins JP, Thompson SG, Deeks JJ, Altman DG. Measuring inconsistency in meta-analyses. BMJ (Clin Res ed). 2003;327:557-60.

18. Begg CB, Mazumdar M. Operating characteristics of a rank correlation test for publication bias. Biometrics. 1994:50:1088-101.

19. Egger M, Davey Smith G, Schneider M, Minder C. Bias in meta-analysis detected by a simple, graphical test. BMJ (Clin Res ed). 1997;315:629-34.

20. Yasuda A, Kimura H, Hayakawa M, Ohshiro M, Kato Y, Matsuura O, et al. Evaluation of cytomegalovirus infections transmitted via breast milk in preterm infants with a real-time polymerase chain reaction assay. Pediatrics. 2003:111:1333-6.
21. Jim WT, Shu CH, Chiu NC, Kao HA, Hung HY, Chang JH, et al. Transmission of cytomegalovirus from mothers to preterm infants by breast milk. Pediatr Infect Dis J. 2004:23:848-51.

22. Mussi-Pinhata MM, Yamamoto AY, do Carmo Rego MA, Pinto PC, da Motta MS, Calixto C. Perinatal or early-postnatal cytomegalovirus infection in preterm infants under 34 weeks gestation born to CMV-seropositive mothers within a high-seroprevalence population. J Pediatr. 2004;145:685-8.

23. Doctor S, Friedman S, Dunn MS, Asztalos EV, Wylie L, Mazzulli T, et al. Cytomegalovirus transmission to extremely low-birthweight infants through breast milk. Acta Paediatr (Oslo, Norway : 1992). 2005;94:53-8.

24. Meier J, Lienicke U, Tschirch E, Krüger DH, Wauer RR, Prösch S. Human cytomegalovirus reactivation during lactation and mother-to-child transmission in preterm infants. J Clin Microbiol. 2005;43:1318-24.

25. Miron D, Brosilow S, Felszer K, Reich D, Halle D, Wachtel D, et al. Incidence and clinical manifestations of breast milk-acquired cytomegalovirus infection in low birth weight infants. J Perinatol. 2005;25:299-303.

26. Omarsdottir S, Casper C, Zweygberg Wirgart B, Grillner L, Vanpée M. Transmission of cytomegalovirus to extremely preterm infants through breast milk. Acta Paediatr (Oslo, Norway : 1992). 2007;96:492-4.

27. Buxmann H, Miljak A, Fischer D, Rabenau HF, Doerr HW, Schloesser RL. Incidence and clinical outcome of cytomegalovirus transmission via breast milk in preterm infants $</=31$ weeks. Acta Paediatr (Oslo, Norway : 1992). 2009;98:270-6.

28. Capretti MG, Lanari M, Lazzarotto T, Gabrielli L, Pignatelli S, Corvaglia L, et al. Very low birth weight infants born to cytomegalovirus-seropositive mothers fed with their mother's milk: a prospective study. J Pediatr. 2009;154:842-8.

29. Jim WT, Shu CH, Chiu NC, Chang JH, Hung HY, Peng CC, et al. High cytomegalovirus load and prolonged virus excretion in breast milk increase risk for viral acquisition by very low birth weight infants. Pediatr Infect Dis J. 2009;28:891-4.

30. Hayashi S, Kimura H, Oshiro M, Kato Y, Yasuda A, Suzuki C, et al. Transmission of cytomegalovirus via breast milk in extremely premature infants. J Perinatol. 2011:31:440-5

31. Mukhopadhyay S, Meyer SA, Permar SR, Puopolo KM. Symptomatic postnatal cytomegalovirus testing among very low-birth-weight infants: indications and outcomes. Am J Perinatol. 2016;33:894-902.

32. Omarsdottir S, Casper C, Navér L, Legnevall L, Gustafsson F, Grillner L, et al. Cytomegalovirus infection and neonatal outcome in extremely preterm infants after freezing of maternal milk. Pediatr Infect Dis J. 2015:34:482-9.

33. Yoo HS, Sung SI, Jung YJ, Lee MS, Han YM, Ahn SY, et al. Prevention of cytomegalovirus transmission via breast milk in extremely low birth weight infants. Yonsei Med J. 2015;56:998-1006

34. Josephson CD, Caliendo AM, Easley KA, Knezevic A, Shenvi N, Hinkes MT, et al. Blood transfusion and breast milk transmission of cytomegalovirus in very low-birth-weight infants: a prospective cohort study. JAMA Pediatr. 2014;168:1054-62

35. Bryant P, Morley C, Garland S, Curtis N. Cytomegalovirus transmission from breast milk in premature babies: does it matter? Arch Dis Child Fetal Neonatal Ed. 2002;87:F75-7.

36. Hamprecht K, Maschmann J, Müller D, Dietz K, Besenthal I, Goelz R, et al. Cytomegalovirus (CMV) inactivation in breast milk: reassessment of pasteurization and freeze-thawing. Pediatr Res. 2004;56:529-35.

37. Schleiss MR. Acquisition of human cytomegalovirus infection in infants via breast milk: natural immunization or cause for concern? Rev Med Virol. 2006;16:73-82.

38. Hamprecht K, Witzel S, Maschmann J, Dietz K, Baumeister A, Mikeler E, et al. Rapid detection and quantification of cell free cytomegalovirus by a highspeed centrifugation-based microculture assay: comparison to longitudinally analyzed viral DNA load and pp67 late transcript during lactation. J Clin Virol. 2003;28:303-16.

39. Anne-Aurélie L, Souad B, Leila K. Clinical findings and autopsy of a preterm infant with breast milk-acquired cytomegalovirus infection. AJP Rep. 2016:6:e198-202

40. You DM, Johnson MD. Cytomegalovirus infection and the gastrointestinal tract. Curr Gastroenterol Rep. 2012;14:334-42.

\section{Publisher's Note}

Springer Nature remains neutral with regard to jurisdictional claims in published maps and institutional affiliations. 